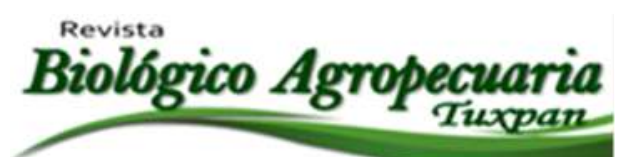

\title{
Un año de observaciones meteorológicas en Loma Bonita, Oax., México; una referencia climatológica para su industria agropecuaria
}

One year of weather observations in Loma Bonita, Oax., Mexico; a climatological reference for its agricultural industry

Soto Molina Víctor H. ${ }^{凶}$, Alanís Méndez José Luis, Pech Canché Juan Manuel

Facultad de Ciencias Biológicas y Agropecuarias, Universidad Veracruzana, 92895, Carretera Tuxpan-

Tampico km 7.5. Col. Universitaria, Tuxpan, Ver., México.

${ }^{凶}$ Autor de correspondencia: islaveracruz@gmail.com

Recibido: 05/08/2019

Aceptado: 23/12/2019

\section{RESUMEN}

A partir de los datos de una estación meteorológica personal se analizaron distintos parámetros atmosféricos en Loma Bonita, Oax., durante el año 2019. Los resultados señalan un rango térmico medio anual de 14.7 a $41.9^{\circ} \mathrm{C}$ y una sensación térmica que va de 14.7 a $57^{\circ} \mathrm{C}$. La menor incidencia de vientos alisios propició una disminución del $44 \%$ en la precipitación total con respecto al valor normal; contrariamente, las temperaturas máximas, mínimas y medias fueron claramente mayores a los valores regulares. Es probable que los cambios observados sean producto de un posible cambio en la climatología de la ciudad, por lo que las actividades agropecuarias de la zona deberían considerar los patrones actuales de temperatura y precipitación. Al mismo tiempo, la salud de la población debe protegerse de los altos niveles de insolación y de radiación UV que se han registrado.

Palabras clave: Clima, climatología agropecuaria, estación meteorológica personal, estadística climatológica, meteorología.

\begin{abstract}
From the data of a personal weather station, different atmospheric parameters were analyzed in Loma Bonita, Oax., Mexico during the year 2019. The results indicate an average annual thermal range of 14.7 to $41.9^{\circ} \mathrm{C}$ and a thermal sensation that goes from 14.7 to $57^{\circ} \mathrm{C}$. A lower incidence of trade winds led to a $44 \%$ decrease in total rainfall compared to the normal value; on the contrary, the maximum, minimum and average temperatures were clearly higher than the regular values. It is possible that the changes observed are the result of a change in the climate of the city, so that agricultural activities in the area should consider current patterns of temperature and precipitation. At the same time, the health of the population must be protected from the high levels of insolation and UV radiation that have been recorded.
\end{abstract}

Keywords: Climate, agricultural climatology, personal weather station, climatological statistic, meteorology. 


\section{INTRODUCCIÓN}

Las condiciones climáticas determinan en gran medida la vocación productiva de una región. El clima, particularmente los índices de temperatura y precipitación, son un factor que rige la productividad agrícola (López, 2015) y ganadera (McCarl, 2010). La variación temporal en cualquiera de estas dos variables les ocasiona efectos negativos, siendo la agricultura la que mayores consecuencias sufra ante el marco del cambio climático (Mendelsohn, 2009), trayendo consigo riesgos alimentarios para la población más vulnerable de América Central (Field et al., 2014).

La región de Loma Bonita, Oax., ha sido un importante productor agropecuario del país. Las características de su clima tropical favorecen el desarrollo de dos principales actividades que representan el sustento económico, directa o indirectamente, de la mayoría de sus habitantes: la producción piñera y de ganado bovino. Durante la década de 1910-1920 se consolida la explotación comercial de piña en Loma Bonita (Uriza-Ávila et al., 2018) y su aportación hasta estos días forma parte del noveno lugar que ocupa México a nivel internacional (Sánchez et al., 2015). Por su parte, la actividad ganadera, si bien no alcanza niveles de importancia similares al del agrícola, su producción la ubica en el sexto sitio del estado (Castro et al., 2015). Castro et al., (2012) señalan que en la región predomina la ganadería de doble propósito, mediante la mezcla de las razas cebú y suizo principalmente.

Tanto la agricultura de piña como la actividad ganadera requieren de ciertos parámetros climáticos para su gestación y desarrollo, así como para lograr las condiciones óptimas de comercialización.
Paralelamente a las propiedades edafológicas y de insolación, e independientemente de la especie de que se trate, en términos generales la piña mexicana requiere de un relieve homogéneo o con pequeños lomeríos que no excedan los $20^{\circ}$ de pendiente, entre el nivel del mar y 900 metros de altitud, así como una temperatura media anual de $\sim 25^{\circ} \mathrm{C}$ y una precipitación anual media acumulada de $\sim 1,500 \mathrm{~mm}$ (Sánchez-Escalante, 2012). De acuerdo con Reyes (1999), las raíces y hojas experimentan su mayor crecimiento entre $30 \mathrm{y}$ $31^{\circ} \mathrm{C}$, siendo nulo con temperaturas inferiores a $21^{\circ}$ y mayores a $35^{\circ} \mathrm{C}$. El máximo desarrollo de la planta ocurre en un rango térmico anual de 24 a $27^{\circ} \mathrm{C}$. La importancia del régimen pluvial radica en que éste se presente a lo largo del ciclo del cultivo. Por otro lado, el ganado bovino requiere de suficiente aporte pluvial tanto para el pastoreo como para su fisiología. En cuanto al factor térmico, los bovinos se adaptan mejor a ambientes templados (Arias et al., 2008), con una media anual térmica $<20^{\circ} \mathrm{C}$, ya que la demanda hídrica aumenta ante el estrés por calor (EC) (Khalifa, 2003). Fox y Tylutki (1998) mencionan que altos niveles de humedad y de temperatura en el aire disminuyen la producción lechera, como consecuencia del EC. El EC es un efecto directo del índice de temperaturahumedad ambiental (ITH). Espinoza et al., (2011) mencionan que las especies lecheras como Holstein y Jersey son las más sensibles a altos niveles de ITH, mientras que las criollas resultan ser más tolerantes. Un ITH menor a 70 mantiene a los bovinos dentro del umbral de confort, mientras que los mayores tienden a ser perjudiciales; el nivel de moderado a grave se ubica por arriba de 80 y el grado de peligro está por arriba de 90 .

Por lo tanto, los episodios de olas de calor veraniegas suelen ser mortales para el ganado (Arias et al., 2008). 
Monitorear las condiciones del tiempo atmosférico y de la climatología de una región que dependa de las actividades del campo como fuente principal de ingresos, es importante para conocer los rangos y su frecuencia, así como los cambios de las principales variables que las integran (Ruíz-Ayala et al., 2018). Por lo que resulta imperativo contar con una estación convencional que registre sistemáticamente los valores diarios de temperatura y precipitación, al menos. Sin embargo, el uso de una estación más completa aportará datos de otras variables con gran valor comercial, ambiental y para la salud humana (OPS, 2012).

En México existen 5,270 estaciones, su ubicación está determinada por las necesidades agrícolas e hídricas de cada región (MirandaAlonso, 2003; 2004), sin embargo de éstas sólo el $62 \%$ están en funcionamiento. Durante la última década se han instalado estaciones meteorológicas automatizadas (EMAs) que monitorean otras variables además de la Temperatura (Temp) y Precipitación (Prec) que se realizan mediante las estaciones convencionales; sus registros de Presión atmosférica (PA), Humedad relativa (HR), Dirección (DV) y Velocidad del viento (VV), y Radiación solar incidente (RS) robustecen el conocimiento atmosférico y eliminan los errores causados por la interpretación y la ausencia del observador, comúnmente identificados en las estaciones convencionales (Arteaga-Ramírez et al., 2017).

Para el caso de Loma Bonita, la Comisión Nacional del Agua tiene registradas dentro de la población dos estaciones que están fuera de servicio (ID 20063 e ID 20237), la primera con un periodo de operación de 1969 a 1983 y la segunda de 1974 a 1985; a diferencia de la primera, la estación 20237 posee datos continuos que permiten obtener promedios normales de precipitación y temperatura. De igual manera, existen estaciones en zonas circundantes que también se encuentran suspendidas. La estación operativa más cercana es la ID 20084 "Papaloapan" a $21 \mathrm{~km}$ al oeste-noroeste de la ciudad con registros a partir de 1947. Por su parte la EMA más próxima está ubicada a $24 \mathrm{~km}$ (estación Ciudad Alemán) también a la misma dirección con relación a la cabecera municipal de Loma Bonita. Considerando que las condiciones atmosféricas para un momento dado dependen, entre otros factores, de la circulación de los vientos y de las fuentes de humedad, es frecuente encontrar diferencias claras de temperatura, de dirección e intensidad de los vientos y de precipitación entre dos sitios con un par de kilómetros de distancia entre sí (Arya, 2001). El caso más notorio está representado por los chubascos vespertinos del verano, que están asociados a nubes convectivas de un punto específico.

En años recientes ha crecido el uso de estaciones meteorológicas personales (EMPs) a nivel mundial, tanto por profesionales como por aficionados de la meteorología. La ventaja de estos dispositivos, más allá del tamaño de $50 \times 20 \times 20 \mathrm{~cm}$ en promedio, radica en el relativamente bajo precio de adquisición; adicionalmente, la facilidad de acceso a los datos en tiempo real por cualquier usuario, vía internet, le otorga un valor excepcional (Butler, 2019). Existen en el mercado distintas marcas y modelos que ofrecen una calidad aceptable y un nivel de precisión muy similar a los equipos sofisticados de mayor costo (Mandement y Caumont, 2019). 
Por la importancia en el conocimiento de las condiciones atmosféricas para las actividades agropecuarias, así como para la salud pública de la región de Loma Bonita, este trabajo está enfocado en analizar un año de observaciones meteorológicas continuas mediante el uso de una EMP instalada en centro de la ciudad. Se pretende que los resultados sirvan como una referencia de las condiciones climatológicas actuales de la ciudad y sus alrededores, para beneficio de agricultores y ganaderos del área; al mismo tiempo, se espera que los datos aportados sean benéficos para el ámbito de la salud pública del municipio.

\section{MATERIALES Y MÉTODOS}

\section{Zona de estudio}

La ciudad de Loma Bonita se localiza al norte del estado de Oaxaca; su límite norte se ubica a $2 \mathrm{~km}$ de la frontera estatal con Veracruz. Es cabecera del municipio con el mismo nombre al que pertenecen 164 localidades de tipo rural. La ciudad posee una población de 21,485 habitantes, albergando el $75.8 \%$ del total municipal (INEGI, 2010) en un polígono aproximado de 3.4 x $3.3 \mathrm{~km}$. Su posición, dentro de la Llanura Costera del Golfo Sur, le determina un relieve plano con una altitud media de 35 msnm, con pequeños lomeríos aledaños que poseen una decena de metros de altura en promedio. Su altitud y la relativa cercanía a la línea de costa condicionan que posea un clima cálido húmedo con lluvias en verano, con una temperatura media anual de $25^{\circ} \mathrm{C}$ y un total acumulado de precipitación media anual de 1,902 mm. El mes más cálido es mayo, con una temperatura media de $34.7^{\circ} \mathrm{C}$; por su parte, enero posee la temperatura menor del año con $16.6^{\circ} \mathrm{C}$ como media. El mes más húmedo es septiembre con un promedio pluvial acumulado de 363.7 $\mathrm{mm}$, mientras que el más seco es marzo con 25.5 $\mathrm{mm}$ de valor promediado (SMN, 2019).

Su geología, en un $83 \%$, está datada en el período Neógeno $\left(23 \times 10^{6}\right.$ años AP) con un lecho rocoso sedimentario, compuesto mayormente por conglomerado en un $61.5 \%$ y arenisca en un 21.6\%; el resto está dividido por lutita y caliza. El suelo es en su mayoría luvisol (51\%), seguido por cambisol (24\%) y vertisol (13\%). Está rodeada por diferentes corrientes perennes: los ríos Obispo, Mangle, Playa Vicente, Tecomate Mixtán, Sesecapan, El Carrizo, El Limón y La Virgen. Dos principales lagos se ubican de manera opuesta en los extremos de la ciudad: la Laguna Cujuliapan al noreste y la Laguna Virginia al suroeste.

La vegetación de sus alrededores está compuesta por pastizales cultivados (51\%), agricultura de temporal $(43 \%)$ y selva baja (4\%); el resto es suelo desnudo (INEGI, 2008). La figura 1 muestra la ubicación de la ciudad. 


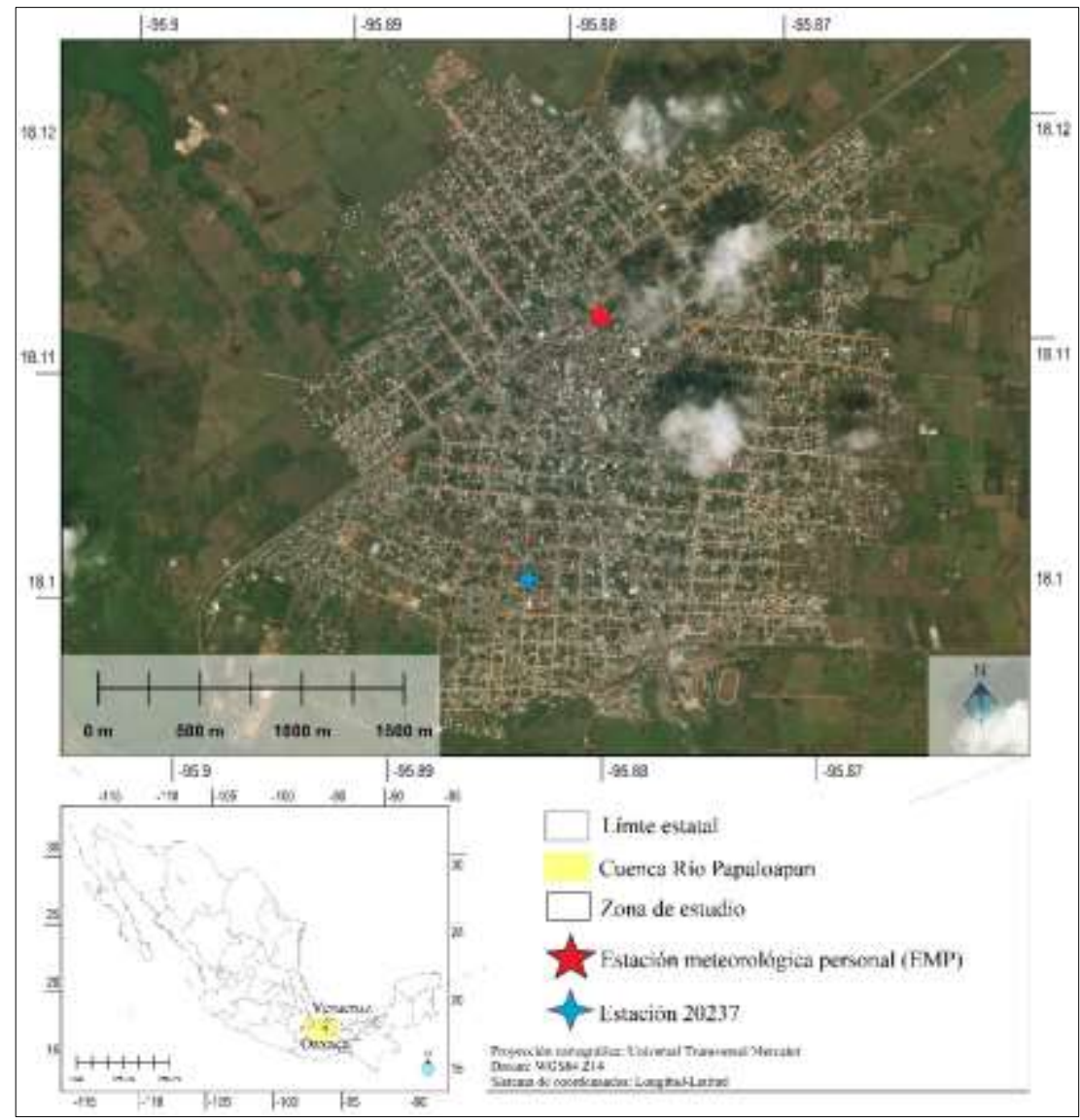

Figura 1. Ciudad de Loma Bonita, Oax. La estrella roja señala la ubicación de la EMP y la azul corresponde a la estación de referencia y comparación.

\section{Equipo utilizado}

Para el registro y almacenamiento de los datos atmosféricos a nivel de superficie se utilizó una EMP nueva, marca Ambient Weather® modelo WS-1002-WiFi (Wireless), integrada con el software WS-1002 V2.5.1. Es alimentada por luz solar y cuenta con baterías "AA" como respaldo. La EMP se instaló en un poste vertical metálico $2 \mathrm{mt}$ por encima de la azotea de un domicilio particular, con coordenadas $18.111^{\circ}$ $\mathrm{N}, 95.879^{\circ} \mathrm{E}$ y a una altitud de $38 \mathrm{msnm}$. La estación fue calibrada desde fábrica para garantizar una precisión aceptable en sus registros. Las especificaciones técnicas de los sensores, según las variables medidas por la EMP se señalan en el cuadro 1. 
Cuadro 1. Variables de medición de la EMP.

\begin{tabular}{|l|l|l|l|}
\hline \multicolumn{1}{|c|}{ Variable } & Rango de operación & Precisión & Resolución \\
\hline Temperatura del aire exterior & -40 a $65^{\circ} \mathrm{C}$ & $\pm 1^{\circ} \mathrm{C}$ & $0.5^{\circ} \mathrm{C}$ \\
\hline Temperatura del aire interior & 0 a $60^{\circ} \mathrm{C}$ & $\pm 1^{\circ} \mathrm{C}$ & $0.5^{\circ} \mathrm{C}$ \\
\hline Humedad relativa exterior & 1 a $99 \%$ & $\pm 5 \%$ & $1 \%$ \\
\hline Humedad relativa interior & 1 a $99 \%$ & $\pm 5 \%$ & $1 \%$ \\
\hline Presión barométrica & 300 a $1100 \mathrm{mb}$ & $\pm 3 \mathrm{mb}$ & $0.3 \mathrm{mb}$ \\
\hline Luz & 0 a $400,000 \mathrm{Lux}$ & $\pm 15 \%$ & $1 \mathrm{Lux}$ \\
\hline Precipitación & 0 a $1000 \mathrm{~mm}$ & $\pm 10 \%$ & $0.03 \mathrm{~mm}$ \\
\hline Dirección del viento & 0 a $360^{\circ}$ & $1^{\circ}$ & $1^{\circ}$ \\
\hline Velocidad del viento & 0 a $160 \mathrm{~km} / \mathrm{h}$ & $\pm 1 \mathrm{~km} / \mathrm{h}$ & $0.1 \mathrm{~km} / \mathrm{h}$ \\
\hline
\end{tabular}

A partir de la combinación de estas variables, el software calcula otros indicadores como la Sensación térmica, Radiación solar, Radiación UV y Punto de rocío. Los datos son almacenados automáticamente por la estación y respaldados en una tarjeta micro SD. La figura 2 muestra la EMP instalada junto con la consola de visualización de los datos en tiempo real. Estos datos pueden ser revisados desde cualquier dispositivo electrónico con acceso a internet mediante

la

página https://www.wunderground.com/dashboard/pws /ILOMABON2. La plataforma de hospedaje de la información de las EMP asigna una clave única para cada estación vinculada, siendo en este caso el ID: ILOMABON2. La plataforma otorga un nivel de calidad y regularidad en el monitoreo de cada estación albergada. La EMP ILOMABON2 ha contado desde el inicio con una medalla dorada, la mayor distinción por calidad y continuidad de servicio, asignada por la plataforma wunderground.com.
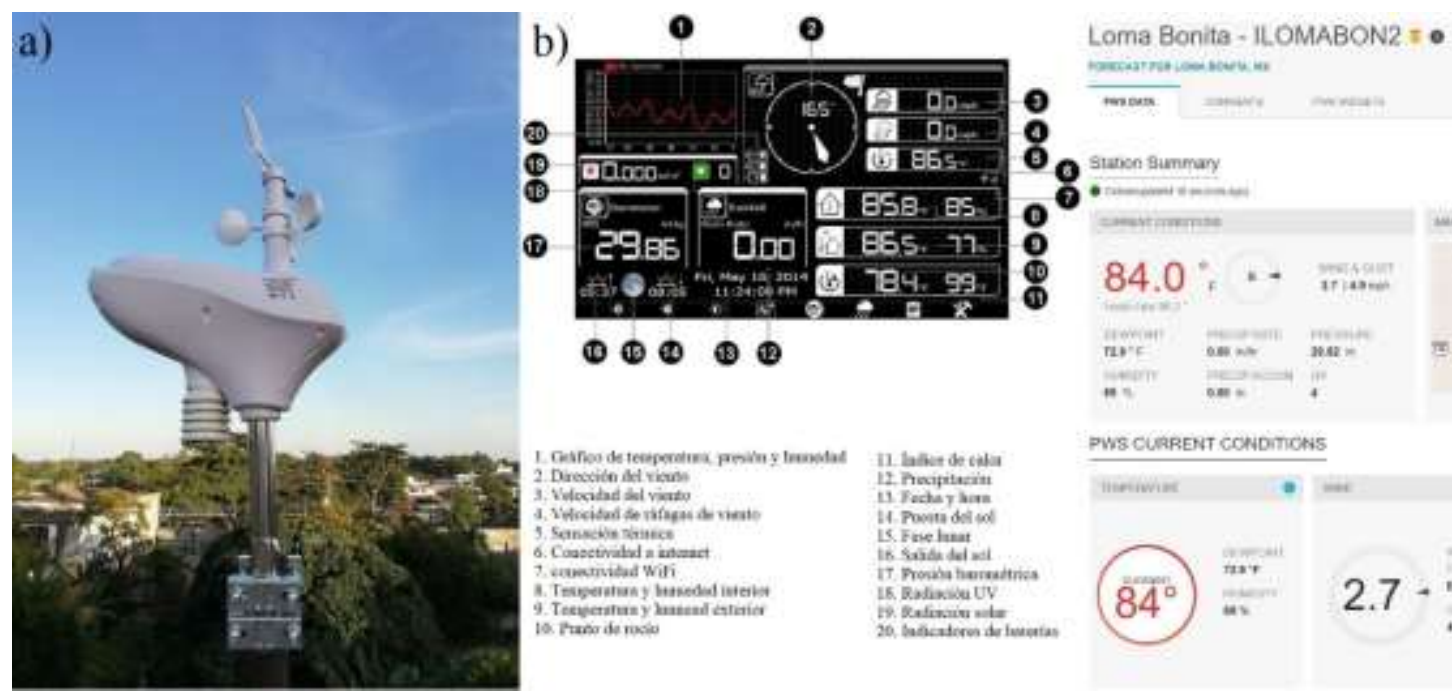

c)

Figura 2. EMP (a) y registros de variables e indicadores atmosféricos en tiempo real (b). La imagen "c" es una captura de pantalla de la página web que alberga la información (temperatura, velocidad, presión y precipitación en ${ }^{\circ} \mathrm{F}$, millas, $\mathrm{mmHg}$ y pulgadas respectivamente). 
Gestión y análisis de los datos

El período de observaciones fue de un año completo de manera regular e ininterrumpida, del 23 de diciembre del 2018 al 22 de diciembre del 2019. Considerando que cada 5 minutos se guardaban los valores registrados, representó 288 registros diarios, lo que al año acumuló 105,120 lecturas para cada una de las variables observadas.

Los datos fueron tabulados en Microsoft $₫$ Excel. Debido a que se trató de un equipo nuevo previamente calibrado y que permaneció en el mismo sitio durante su funcionamiento, con observaciones ininterrumpidas, se consideró que los datos registrados eran estadísticamente homogéneos, representando las condiciones atmosféricas reales en cada momento (OMM, 2011; Firat et al., 2012). No obstante, mediante análisis de tipo exploratorio y gráfico se realizaron pruebas de inconsistencia entre los valores de temperatura y precipitación (Castro y Carvajal-Escobar, 2010), verificando que la Temperatura máxima (Tmáx) fuera mayor que la mínima diaria (Tmin) y que los datos de las variables restantes no tuvieran valores negativos. Posterior a este proceso se verificó la inexistencia de valores atípicos (outliers) considerando que para cada una de las series de datos los valores extremos se mantuvieran en un máximo de 4 veces el valor de la desviación estándar (Vea et al., 2012). En el caso de la precipitación (Prec), los valores atípicos fueron ocasionados por tormentas intensas durante el verano, justificando así el excedente de la desviación típica.

Para todas las variables se calcularon los promedios diarios y mensuales. Cada uno de los periodos fueron graficados $\mathrm{y}$ se realizaron análisis de frecuencias, también se calculó el ITH (Valtorta y Gallardo, 1996; Saravia et al., 2002). Finalmente los valores de temperatura y precipitación se compararon con las normales climatológicas de la estación 20237.

\section{RESULTADOS Y DISCUSIÓN}

Los gráficos de la figura 3 muestran las series temporales de las principales variables observadas. En ella se aprecia que los gráficos de área corresponden a las fluctuaciones de cada variable, mientras que las líneas continuas representan los valores medios correspondientes. 


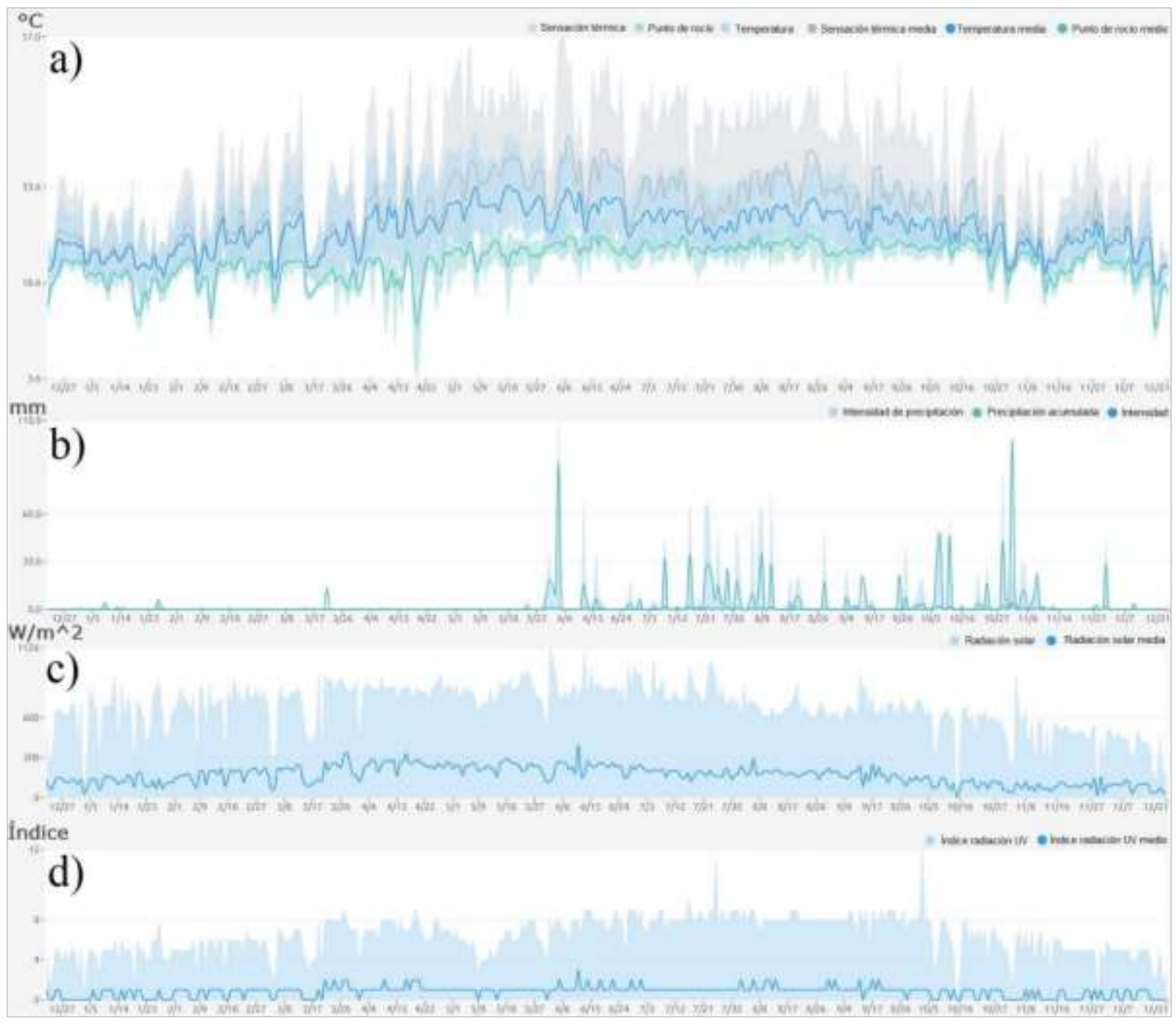

Figura 3. Series temporales principales. La gráfica "a" muestra los indicadores asociados a la variable temperatura; la "b" corresponde a la precipitación; la "c" a la radiación solar y la "d" al índice de radiación $U V$.

Por su parte, la figura 4 muestra las gráficas de cada una de las variables térmicas. frecuencias de los valores correspondientes a 

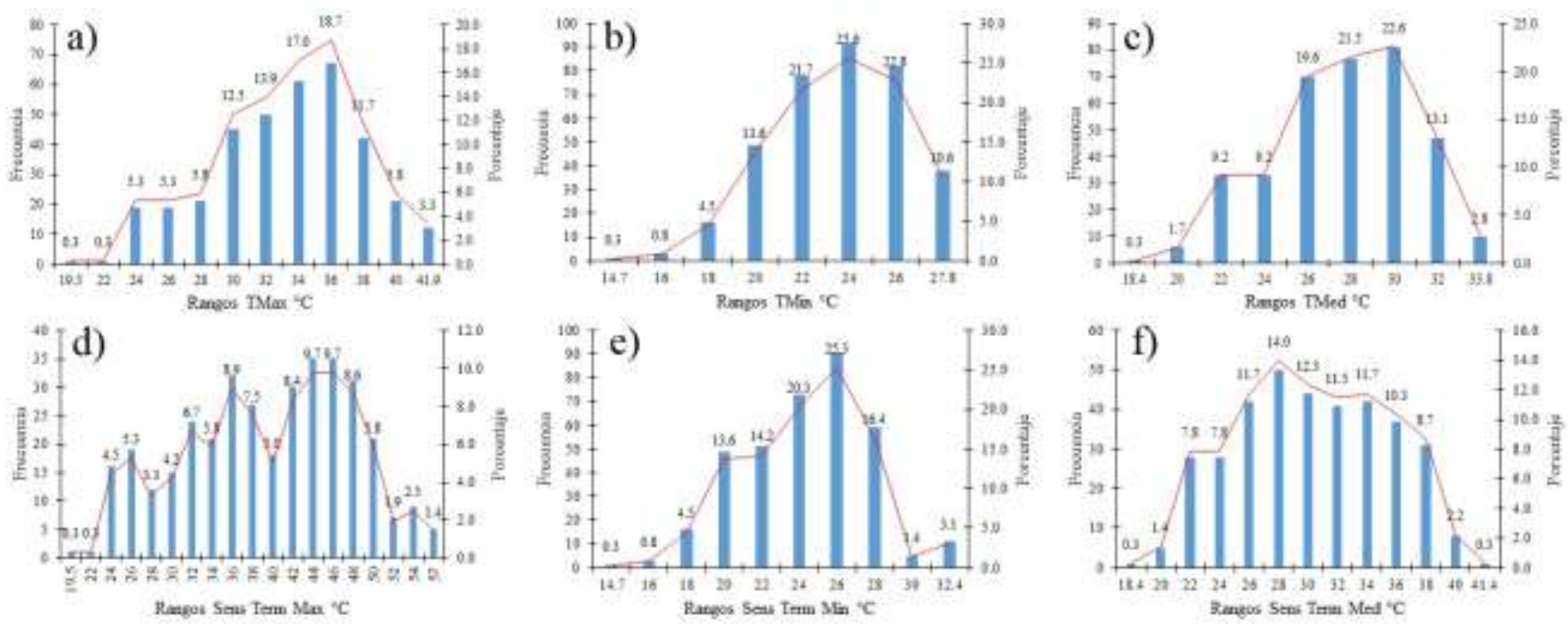

Figura 4. Frecuencia de valores de temperatura máxima (a), mínima (b) y media (c); así como la sensación térmica máxima (d), mínima (e) y media (f).

Con base en la secuencia de gráficas de la figura 4 se observa que la Tmáx más frecuente alcanza valores entre 34 y $36^{\circ} \mathrm{C}$, siendo este último el más recurrente con el $18.7 \%$ de las veces; poco más de una decena de ocasiones se han alcanzado $41.9^{\circ} \mathrm{C}$. La Tmin más común está en el rango de 22 a $24^{\circ} \mathrm{C}$ con una frecuencia del 25.6\%; puede observarse que la Tmin registrada fue de $14.7^{\circ} \mathrm{C}$ (figura $4 \mathrm{~b}$ ). Finalmente, la Tmed más común se ubica en $30^{\circ} \mathrm{C}$ durante el $22.6 \%$ del año (figura 4 c). La sensación térmica, una combinación de las variables de temperatura, humedad y velocidad del viento, muestra un patrón muy semejante al correspondiente de la temperatura. Durante el verano, es más frecuente que la sensación máxima alcance los 44 y $46^{\circ} \mathrm{C}$, aunque se aprecian registros de hasta $57^{\circ} \mathrm{C}$ (figura $4 \mathrm{~d}$ ). La sensación térmica mínima alcanza $26^{\circ} \mathrm{C}$ el $25.3 \%$ de las veces; el valor de $14.7^{\circ} \mathrm{C}$ durante el invierno sugieren la ausencia de viento durante el momento de mínima temperatura (figura 4 e). Por último, la sensación térmica media más frecuente es $28^{\circ} \mathrm{C}$ el $14 \%$ del año; se observan valores que van desde 18.4 a $41.4^{\circ} \mathrm{C}$ (figura $4 \mathrm{f}$ ).

Los valores correspondientes a la variable del viento pueden observarse en la figura 5. En la gráfica y el cuadro incluido se aprecia la dirección y su frecuencia, la velocidad media del viento y de las ráfagas. 


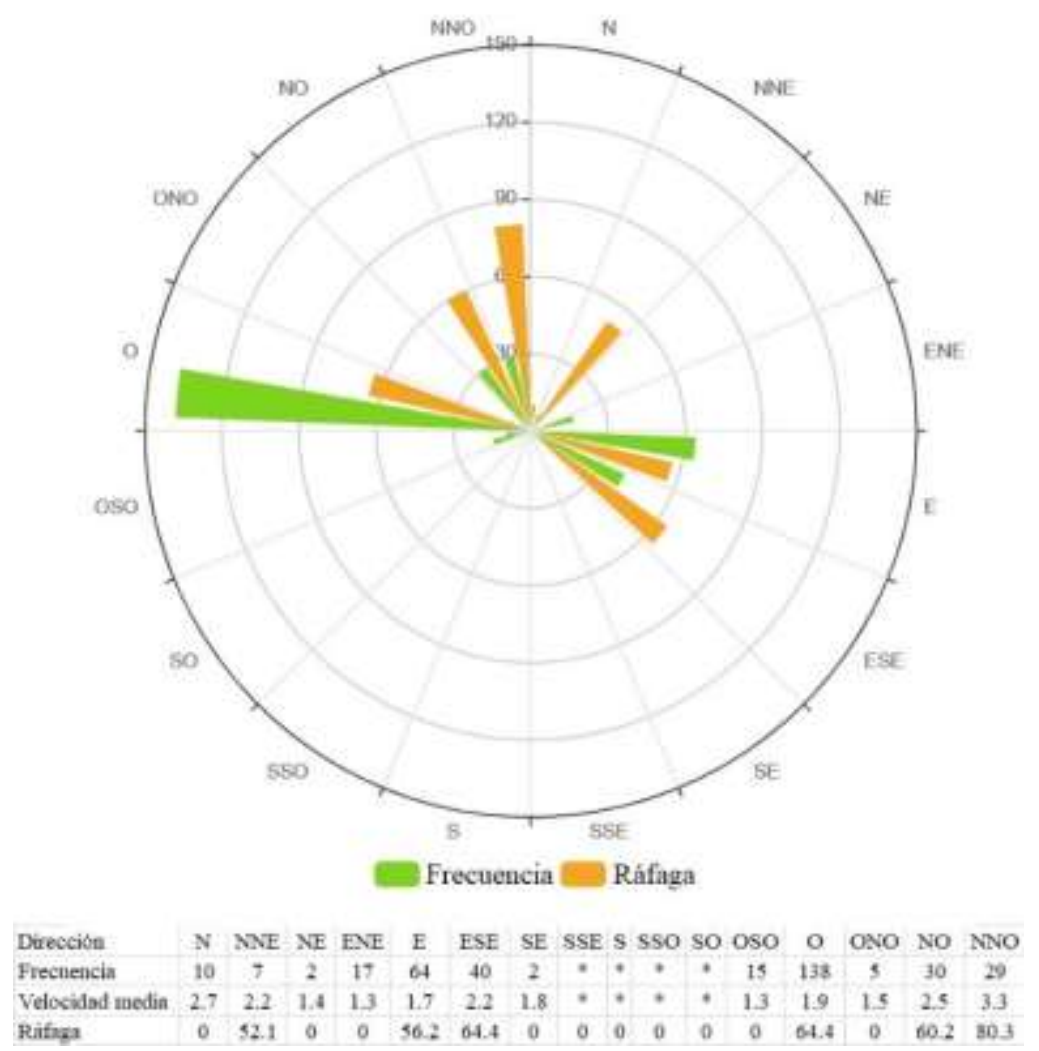

Figura 5. Rosa de los vientos con frecuencia, velocidad e intensidad de las ráfagas. Los asteriscos señalan que el viento procedente de la dirección correspondiente fue imperceptible.

La figura 5 muestra que durante el año, 138 días el viento procedió del oeste $(\mathrm{O})$, siendo por mucho, la dirección más frecuente, mientras que el procedente del este registró una frecuencia de 64. Se puede observar que la velocidad media del viento fluctúa entre 1.3 y $3.3 \mathrm{~km} / \mathrm{h}$, siendo el menor valor del oeste-suroeste (OSO) y el mayor del nor-noroeste (NNO). Es notorio que no obstante de tratarse del valor medio anual, la incidencia de los "nortes" durante la parte final del otoño, invierno y principio de la primavera, influyen en este parámetro. Esto queda de manifiesto en las ráfagas registradas; del NNO se registraron velocidades de $80.3 \mathrm{~km} / \mathrm{h}$. Las "suradas" del verano (ESE) registraron velocidades de hasta $64.4 \mathrm{~km} / \mathrm{h}$; es probable que los registros de mayor temperatura y sensación térmica fueran producto de estas oleadas de calor. Resulta oportuno señalar que los alisios (E), comúnmente presentes entre mayo y octubre, no tuvieron gran ocurrencia durante el año. Este aparente debilitamiento de los alisios sirvió para que se registrara una mayor incidencia de la corriente del oeste (Jáuregui, 1975). Debido a que el mayor índice pluvial del año es producto de las lluvias del verano y otoño, consecuencia de la incidencia de los alisios (Jáuregui, 2003), su debilitamiento condicionó la baja acumulación pluvial registrada durante el año de estudio, la cual inició a finales de mayo con pocos picos de acumulación hasta la parte final de octubre (figura 3 b). La figura 6 señala los valores mensuales de precipitación: 


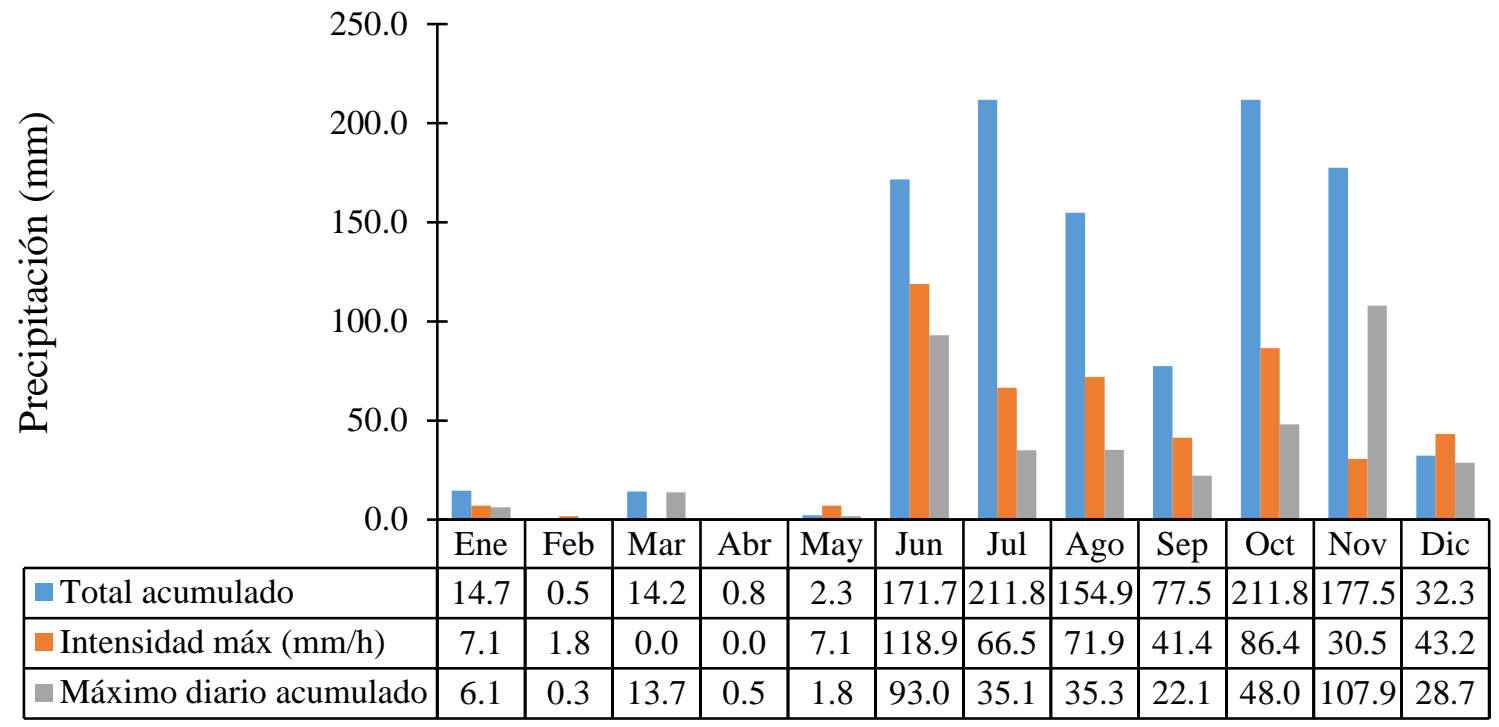

Figura 6. Precipitación mensual acumulada con valores máximos de intensidad y de acumulación máxima diaria.

Se observa una precipitación anual acumulada de 1,070 mm, muy baja en comparación a los 1,902.3 mm que la estación 20237 muestra como valor medio entre los años 1974-1985. No obstante, la lluvia sigue estando presente entre el verano y el otoño. Octubre ocupa el primer sitio con $211.8 \mathrm{~mm}$ y febrero fue el mes más seco con $0.5 \mathrm{~mm}$ totales, muy semejante con abril. Contrariamente, la tormenta convectiva del 4 de junio registró una intensidad máxima de 118.9 $\mathrm{mm} / \mathrm{h}$, tal como se observa también en la figura 3 (b), siendo la máxima tasa del año. Por su parte, el valor máximo de lluvia diaria acumulada se presentó el 1 de noviembre con $107.9 \mathrm{~mm}$ representando la más alta del año.

Con respecto a la radiación solar, la figura 7 señala los valores registrados durante el año.

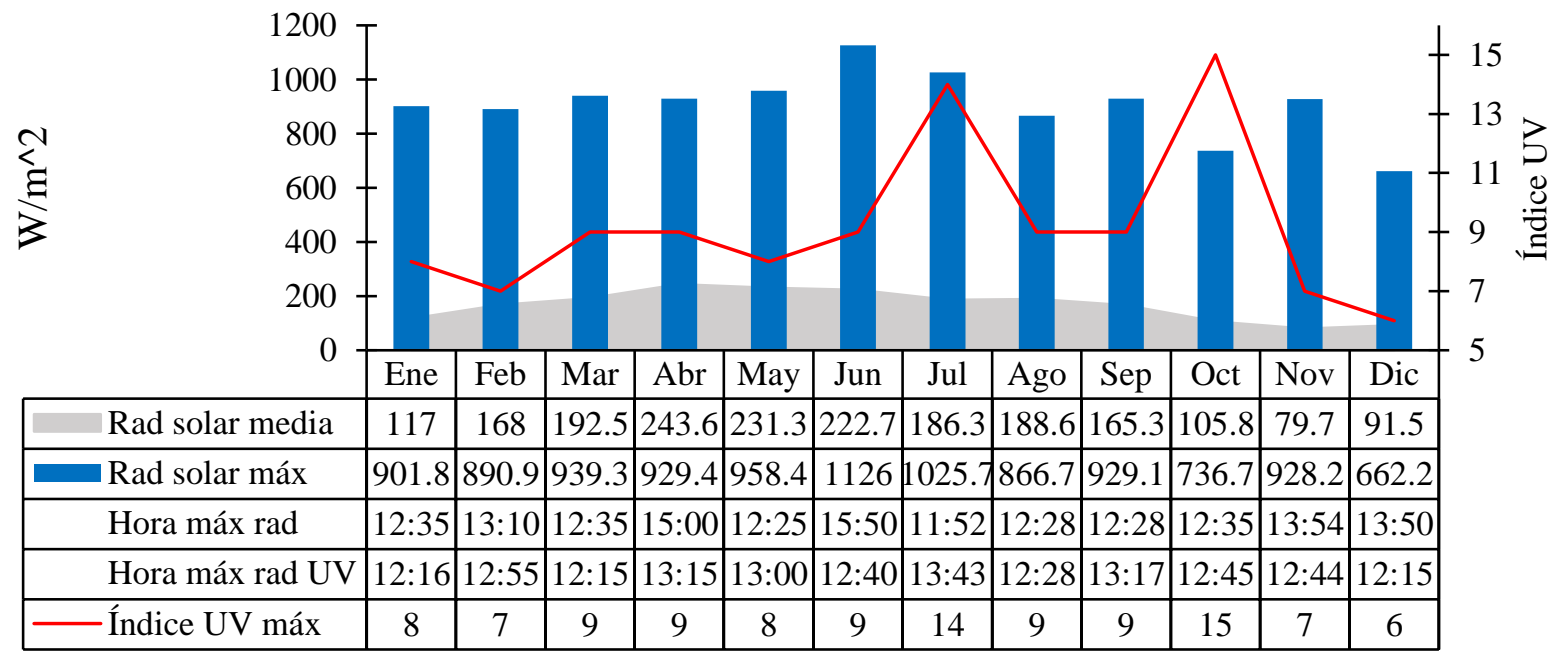

Figura 7. Valores de radiación solar e índice UV. 
Durante abril se registraron $243.6 \mathrm{~W} / \mathrm{m}^{2}$ como valor medio, siendo la cresta de la aparente parábola de acumulación de radiación solar a lo largo del año. Es importante mencionar que gran parte de este parámetro depende del grado de cobertura nubosa y de su espesor; por lo que las nubes convectivas del verano y otoño tienden a bloquear con mayor eficacia la radiación solar; de esta manera, junio y julio deberían de registrar un máximo de radiación acumulada con un cielo totalmente despejado. Lo anterior queda ratificado con el valor de 1,126 y $1,025.7 \mathrm{~W} / \mathrm{m}^{2}$ para el 1 de junio y 5 de julio respectivamente (ver también figura 3 “c"). La máxima insolación se observa entre las 12:00 y 16:00 hrs. El índice de radiación UV no muestra una coincidencia estacional, ya que se aprecian valores máximos de 14 y 15 durante el 14 en julio y 3 de octubre respectivamente, siendo los picos del año. Lo resaltable del caso, es que el horario de máximo índice UV se presenta entre las 12:00 y 14:00 del día (ver también figura 3 "d"), independientemente del mes en que se registre. Con excepción de noviembre y diciembre, que registraron valores "altos" de 6 y 7, el resto del año alcanzó un índice "muy alto", entre 8 y 10; más aún, en julio y octubre se registraron valores "extremos" de 14 y 15 respectivamente. La importancia de estos parámetros, radica en que a partir del nivel 11 se considera "altamente peligroso" para la salud humana (Cañarte, 2010).

Los índices de ITH, producto de la combinación de la temperatura y la humedad relativa máximas que fueron calculados, junto con su frecuencia se muestran en la figura 8:

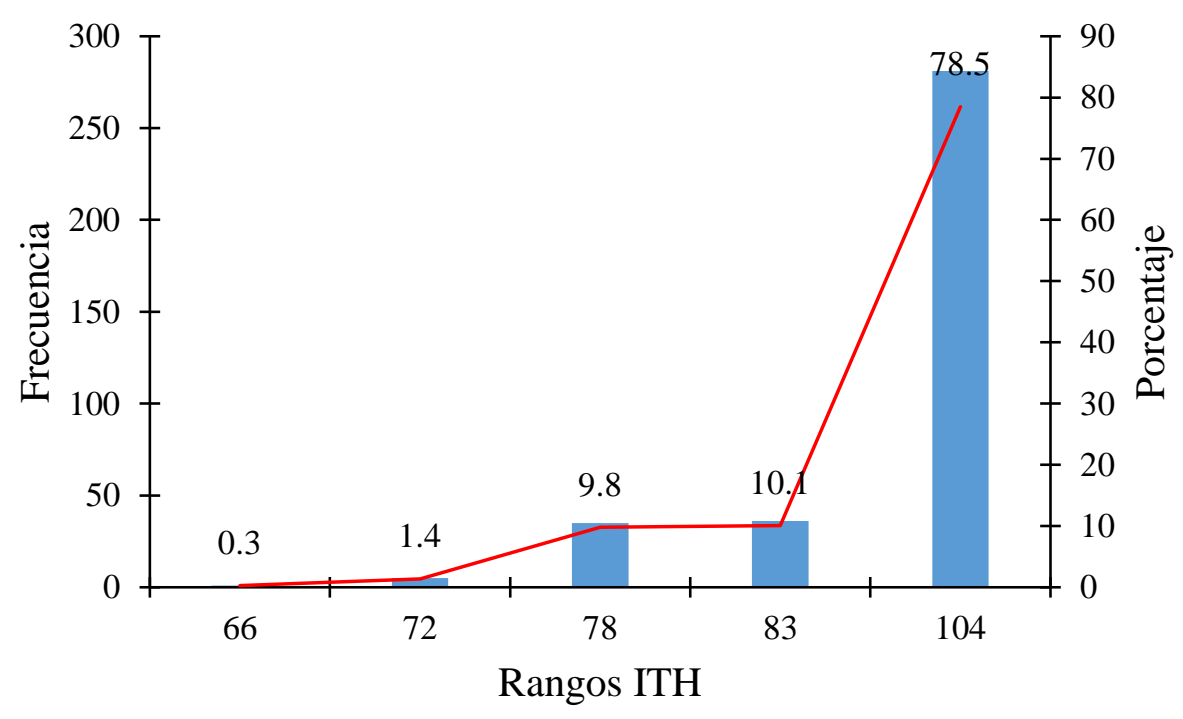

Figura 8. Índices ITH y frecuencia con base en la temperatura y humedad relativa máximas diarias.

De acuerdo con los parámetros de Valtorta y Gallardo (1996) y Saravia et al., (2002), el $88.6 \%$ del año se registró en algún momento un ITH que va de moderado a grave $(10.1 \%)$ hasta niveles peligrosos (78.5\%). Estas condiciones de estrés bovino pudieron representar consecuencias negativas en su fisiología y producción, así como un alto potencial de riesgo 
mortal en algún momento de los 281 días en que alcanzaron los máximos niveles de ITH.

Por último, si se comparan los datos del 2019 contra los valores medios de la estación 20237 para el periodo 1974-1985 en que estuvo operando, los valores medios mensuales correspondientes se pueden contrastar en la figura 9:

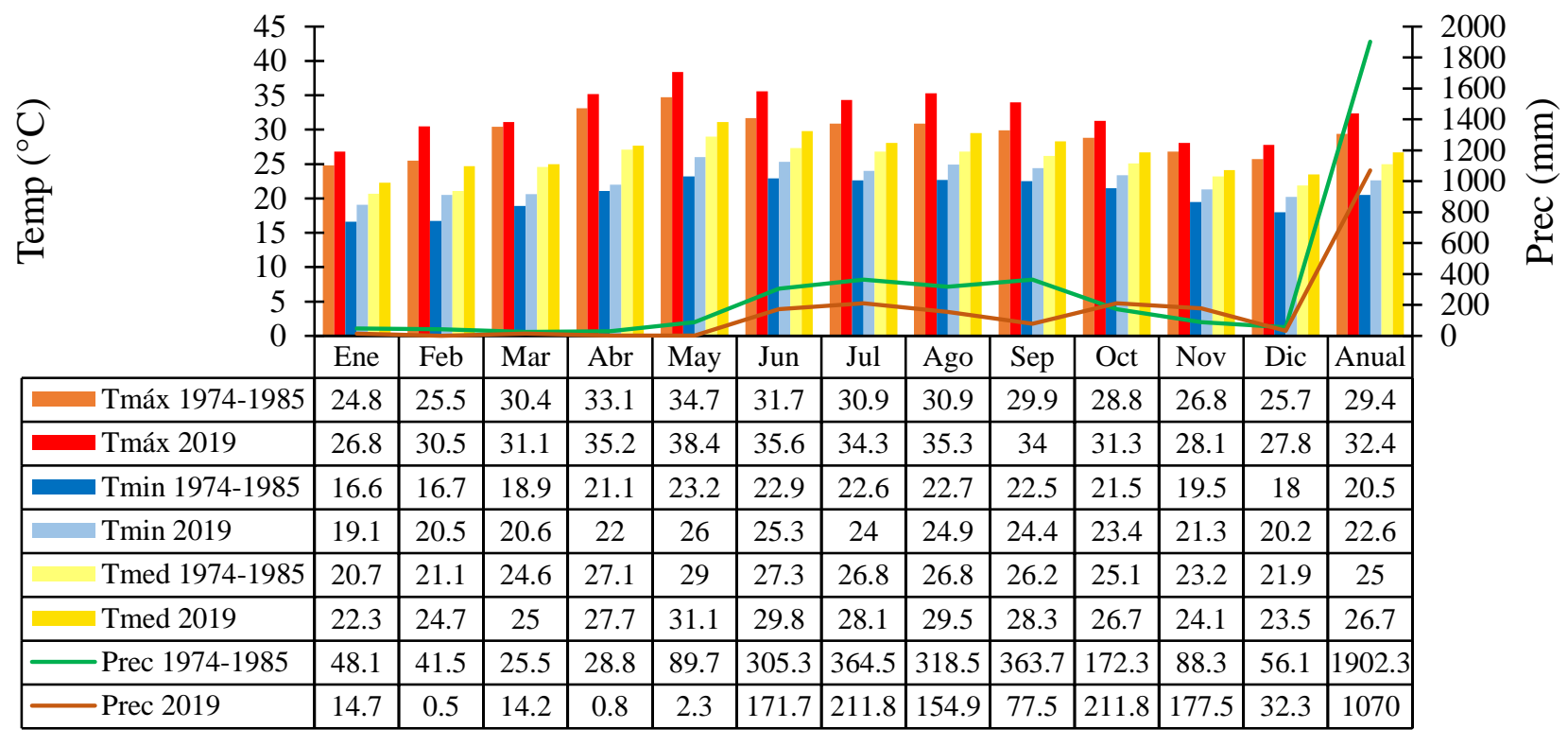

Figura 9. Comparación de medias mensuales entre 2019 y el período 1974-1985.

No existe duda en cuanto al aumento de la temperatura para cada mes con respecto a 34 años atrás, y por lo tanto también para la media anual, al menos durante el 2019. La Tmáx anual aumentó de 29.4 a $32.4^{\circ} \mathrm{C}$, la Tmin pasó de 20.5 a $22.6^{\circ} \mathrm{C}$ y la Tmed de $25^{\circ} \mathrm{C}$ pasó a ser de $26.7^{\circ} \mathrm{C}$ como promedio anual. Por su parte, la precipitación total del año de estudio muestra una marcada disminución del $44 \%$ con respecto al valor normal de 1974-1985. Es probable que estas diferencias obedezcan en gran medida a un posible cambio en la climatología de Loma Bonita, o por lo menos, se trató de un año muy por encima de los valores normales de temperatura y muy por debajo del nivel de precipitación de años anteriores. Aun tratándose sólo de un año de observaciones, no se puede omitir pensar que los patrones de variabilidad climática que se están experimentando a escala global estén también presentándose en la zona de estudio. Los cambios observados con este trabajo obligan a reconsiderar la toma de decisiones en cuanto al cultivo de la piña de la región, así como con la producción de ganado bovino; ya que para el primer caso, la temporalidad de plantación, fertilización y fumigación, así como la planeación de la cosecha puede estar modificándose a través del tiempo. Para el caso de la ganadería, los periodos de crianza y pastoreo pueden verse alterados en función del volumen pluvial; por su parte, las oleadas de calor veraniego requieren de mayores consideraciones para prevenir el estrés hídrico y calórico, así como la posible muerte de cabezas de ganado. 


\section{CONCLUSIONES}

2019 fue un año que registró temperaturas mínimas de 14.7 y máximas de $41.9^{\circ} \mathrm{C}$. Esta variable, aunada a la ausencia de viento mientras se registraba el valor mínimo, condicionó que la sensación térmica mínima obtuviera el mismo valor. Por el contrario, combinada con el contenido de humedad atmosférica derivó en una sensación térmica máxima de $57^{\circ} \mathrm{C}$. La temperatura media, por su parte, se conservó mayormente alrededor de los $30^{\circ} \mathrm{C}$ durante el año. Los vientos alisios del este fueron menos frecuentes que las corrientes del oeste; esta diferencia debió influir en el bajo índice de precipitación acumulada que se registró durante el año. Mientras que la velocidad promedio del viento rondó los $2 \mathrm{~km} / \mathrm{h}$, las ráfagas ocasionadas por los sistemas frontales del otoño e invierno alcanzaron los $80.3 \mathrm{~km} / \mathrm{h}$. Por su parte, se observa que la radiación solar más intensa comienza con la primavera y alcanza mayores valores durante el verano entre las 12:00 y 16:00 hrs. La radiación UV alcanzó el índice 15, muy por encima del nivel considerado como peligroso para la salud humana; las horas de mayor intensidad fueron entre las 12:00 y 14:00. Estos picos no están estrictamente relacionados con la alta insolación veraniega.

Los altos niveles de ITH registrados pudieron repercutir en la diminución del metabolismo del ganado bovino y su producción lechera; pero sobre todo, le pudieron representar un serio peligro de muerte, como consecuencia de las ondas de calor combinadas con un alto contenido de humedad atmosférica.

Con respecto al promedio mensual y anual de temperatura, tanto la Tmáx, Tmin y Tmed mostraron valores claramente superiores comparados con los correspondientes entre los años 1974-1985. La precipitación acumulada, por su parte, fue $44 \%$ menor que los valores normales del mismo periodo. Es probable, por lo tanto, que se estén registrando cambios en las condiciones climáticas de la ciudad; esta variabilidad mostrada, posiblemente obligue a tomar nuevas medidas en cuanto a los procesos de la agricultura y ganadería propios de la región. Del mismo modo, resulta imperativo considerar con la seriedad necesaria estos resultados para minimizar en lo posible, los riesgos asociados a la salud de la población.

\section{LITERATURA CITADA}

Arteaga-Ramírez, R., Cervantes-Osornio, R., Vázquez Peña, M. A., y Ojeda Bustamante, W. (2017). Estación meteorológica convencional versus automática sus diferencias evaluadas con índices estadísticos Chapingo, México. Revista mexicana de ciencias agrícolas, 8(6), 1301-1314.

https://doi.org/10.29312/remexca.v8i6.29

Arias, R. A., Mader, T. L., y Escobar, P. C. (2008). Factores climáticos que afectan el desempeño productivo del ganado bovino de carne y leche. Archivos de medicina veterinaria, 40(1), 7-22. https://doi.org/10.4067/S0301-732X2008

Arya, P. S. (2001). Introduction to micrometeorology (Vol. 79). Academic press $420 \mathrm{pp}$.

Butler, M. K. (2019). Personal weather stations and sharing weather data via the Internet. Weather, 74(1), 22-29. https://doi.org/10.1002/wea.3206

Cañarte, S., K. (2010). Radiación ultravioleta y su efecto en la salud. Ciencia UNEMI, 26-33.

Castro, L. M., y Carvajal Escobar, Y. (2010). Análisis de tendencia y homogeneidad de series climatológicas. Ingeniería de Recursos Naturales y del Ambiente, (9), 15-25.

Castro, C. J. M., Rivera, J. C., y Zavaleta, J. A. (2012). Características de la producción 
y comercialización de leche bovina en sistemas de doble propósito en Dobladero, Veracruz. Revista Mexicana de Agronegocios, 30, 816-824.

Castro, C. J. M., Rivera, J. C., Merales, O. L. A., Forsythe, E. D., \& Cruz, M. T. K. (2015). Agentes y márgenes de comercialización del ganado bovino para abasto en Loma Bonita, Oaxaca. Revista Mexicana de Agronegocios, 36, 1188-1198.

Espinoza, J., Ortega, R., Palacios, A., y Guillén, A. (2011). Tolerancia al calor y humedad atmosférica de diferentes grupos raciales de ganado bovino. Revista MVZ Córdoba. 16(1), 2302-2309. https://doi.

Field, C. B., Barros, V. R., Dokken, D. J., Mach, K. J., Mastrandrea, M. D., Bilir, T. E., y White, L.L. (Eds.). (2014). IPCC, 2014: Climate Change 2014: Impacts, Adaptation, and Vulnerability. Part A: Global and Sectoral Aspects. Contribution of Working Group II to the Fifth Assessment Report of the Intergovernmental Panel on Climate Change. Cambridge University Press, Cambridge, United Kingdom and New York, NY, USA. https://doi.org/10.1017

Firat, M., Dikbas, F., Koc, A. C., \& Gungor, M. (2012).Analysis of temperature series: estimation of missing data and homogeneity test. Meteorological Applications, 19(4), 397-406. https://doi

Fox, D. G. \& Tylutki, T. P. (1998). Accounting for the effects of environment on the nutrient requirements of dairy cattle. $J$ Dairy Sci 81, 3085-3095. https://doi.org Instituto Nacional de Estadística Geografía e Informática, INEGI. (2008). Prontuario de información geográfica municipal de los Estados Unidos Mexicanos. Loma Bonita, Oaxaca, 20004.
Instituto Nacional de Estadística Geografía e Informática, INEGI. (2010). Censo de Población y Vivienda.

Jáuregui O., E. (1975). Los sistemas de tiempo en el Golfo de México y su vecindad. Investigaciones geográficas, (6), 7-36. https://doi.org/10.14350/rig.58 Jáuregui O., E. (2003). Algunos conceptos modernos sobre la circulación general de la atmósfera. Investigaciones geográficas, (50), 121-143.

Khalifa H., H. (2003). Bioclimatology and adaptation of farm animals in a changing climate. In: Interactions between climate and animal production. Proc Symp, EAAP Technical series $\mathrm{N}^{\circ}$ 7, 15-29.

López, A. (2015). Cambio climático y actividades agropecuarias en América Latina. Estudios del Cambio Climático en América Latina. Santiago: Comisión Económica para América Latina y el Caribe (CEPAL). 76 pp.

Mandement, M., \& Caumont, O. (2019, July). Contribution of Personal Weather Stations to the Observation of DeepConvection Features Near the Ground. In 18th Conference on Mesoscale Processes. AMS. https://doi.org/10.5194

McCarl, B. A. (2010). Analysis of climate change implications for agriculture and forestry: an interdisciplinary effort. Climatic Change, 100(1), 119-124. https

Mendelsohn, R. (2009). The impact of climate change on agriculture in developing countries. Journal of Natural Resources Policy Research, 1(1), 5-19. https://doi.o Miranda-Alonso S. (2003). Actualización de la base de datos climatológica CLICOM (1989-2002) para la cuenca Lerma Chapala. Informe Técnico TH-0335. IMTA. 
Miranda-Alonso S. (2004). Manejador de datos climatológicos en Excel. AMH, XVIII Congreso Nacional de Hidráulica, San Luis Potosí, S. L. P.

Organización Meteorológica Mundial, OMM. (2011). Guía de prácticas climatológicas No. 100.ISBN 978-92-63-30100-0. Ginebra, Suiza. 128 pp.

Organización Panamericana de la Salud, OPS. 2012. Proteger la salud frente al cambio climático: evaluación de la vulnerabilidad y la adaptación. Washington, DC: OPS. 80 pp.

Reyes, R. 1999. Manual técnico: Buenas prácticas en el cultivo de la piña. Panamá. Consultado 12 de diciembre $2019 . \quad$ Disponible en http://www.oirsa.org/aplicaciones/subid oarchivos/BIBLIOTECAVIRTUAL/M ANUALPIÑA.PDF.

Ruiz-Ayala, D. C., Vides-Herrera, C. A., \& Pardo-García, A. (2018). Monitoreo de variables meteorológicas a través de un sistema inalámbrico de adquisición de datos. Rev. investig. desarro. innov, 8(2), 333-341.https://doi.org/10.19053/202783 06.v8.n2.2018.7971

Sánchez, M., Ahuja, S., y Acevedo, R. (2015). Producción de Piña Cayena Lisa y MD2 (Ananas comosus L.) en condiciones de Loma Bonita, Oaxaca. En E. Figueroa, L. Godínez, F. Pérez (eds.) Ciencias de la Biología y Agronomía. Handbook T-I. CECORFAN, Texcoco de MoraMéxico.
Sánchez Escalante, J. A. (2012). Manual para la producción de una piña de calidad. 44 pp. Saravia, C., Cruz, G., y Franco, J. (2002). Cálculo del Índice de Temperatura y Humedad diario y su estimación a partir de un mínimo de registros. In Congreso Argentino de Agrometeorología. Anales. AADA. Córdoba, Argentina.

Servicio Meteorológico Nacional, SMN. (2019). Normales climatológicas. Recuperado de https://smn.conagua.gob.mx/tools/RES OURCES/Normales5110/NORMAL202 37.TXT fecha de consulta 10 diciembre del 2019.

Uriza- Ávila, D. E., Torres- Ávila, A., Aguilar- Ávila, J., Santoyo- Cortés, V. H., Zetina- Lezama, R., y RebolledoMartínez, A. (2018). La piña mexicana frente al reto de la innovación. Avances y retos en la gestión de la innovación. Colección Trópica Húmeda. Chapingo, Estado de México. México: UACh. 479 pp.

Valtorta, S., Gallardo, M. (1996). El estrés por calor en producción lechera. En Instituto Nacional de Tecnología Agropecuaria. Argentina. Miscelánea $N^{\circ}$ 81, 173-185.

Vea, P. E., Duran, M. P., y Aguilar, E. (2012). Tendencias recientes e índices de cambio climático de la temperatura y la precipitación en Andorra, Pirineos (1935-2008). Pirineos, 167, 87-106. https://doi.org/10.3989/Pirineos.2012.16 7005 


\section{Copyright (c) 2019 Victor H. Soto Molina, José Luis Alanis Méndez y Juan Maruel Pech Canché}

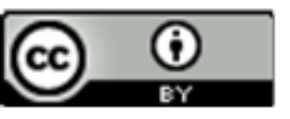

Este tex to está protegido por una licencia licencia CreativeCommons $\underline{4.0}$

Usted es libre para Compartir —copiar y redistribuir el material en cualquier medio o form ato-y Adaptar el documento —remezclar, transformar y crear a partir del material一 para cualquier propósito, incluso para fines comerciales, siempre que cumpla la condición de:

Atribuci ón: Usted debe dar crédito a la obra original de manera adecuada, proporcionar un enlace a la licencia, e indicar si se han realizado cam bios. Puede hacerlo en cualquier form a razonable, pero no de form a tal que sugiera que tiene el apoyo del licenciante olo recibe por el uso que hace de la obra.

Resumendelicencia - Textocompletodelalicencia 